\title{
The Importance of Frameworks for Directing Empirical Questions: Reply to Goodie and Fantino (2000)
}

\author{
Marsha C. Lovett \\ Carnegie Mellon University
}

\author{
Christian D. Schunn \\ George Mason University
}

\begin{abstract}
A. S. Goodie and E. Fantino (2000) make two main criticisms of the predictions of M. C. Lovett and C. D. Schunn's (1999) RCCL model. (RCCL is pronounced "ReCyCLe"; it stands for Represent the task, Construct a set of action strategies, Choose from among those strategies according to success rate, Learn new success rates.) In both cases, the authors believe the criticisms reflect a failure to appreciate the difference between broad frameworks and specific mathematical/computational models. In this article, the value of a broad framework, such as RCCL, in directing new empirical analyses and guiding theoretical development is shown. In particular, RCCL expands on existing work to reveal how variability and change in mental representations influence base-rate sensitivity. The authors also address several other issues raised by A. S. Goodie and E. Fantino (2000) and show that qualitative shifts in individuals' choice behavior are present in their original data-a key prediction of RCCL that does not appear in previous accounts.
\end{abstract}

Goodie and Fantino (2000) make two main criticisms of the predictions of Lovett and Schunn's (1999) RCCL framework (RCCL is pronounced "ReCyCLe"; it stands for Represent the

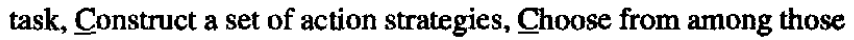
strategies according to success rate, Learn new success rates.). The first is about whether RCCL makes novel predictions. The second is that RCCL's specification is not constrained enough to generate specific predictions. We believe both of these criticisms stem from a failure to appreciate the difference between broad frameworks and specific mathematical or computational models. RCCL was proposed as a broad framework, not as a specific computational model, and should not be criticized for missing characteristics of a specific model (i.e., very precise predictions or unique coverage). In addition, several of Goodie and Fantino's comments reflect a fundamental misunderstanding of the role of base rates in RCCL, in particular, how RCCL captures individuals' propensity to learn the base rates of a variety of different task features.

In this response, we first review the novel features of RCCL (including some that Goodie and Fantino acknowledge in their commentary) and show how RCCL expands on existing theory by incorporating representational change and individual differences between participants that go beyond mere sampling variability. Second, we argue that using a broad framework such as RCCL is valuable because it raises new empirical questions, unifies and expands existing theories, and offers a conceptual foundation for building specific computational models. Finally, we again try to clarify how RCCL's strategy-based learning leads to different

Correspondence concerning this article should be addressed to Marsha C. Lovett, Department of Psychology, Carnegie Mellon University, Pittsburgh, Pennsylvania 15213, or to Christian D. Schunn, Department of Psychology, MSN 3F5, George Mason University, Fairfax, Virginia 22030-4444. Electronic mail may be sent to either lovett@cmu.edu or schunn@gmu.edu. kinds of base-rate sensitivity depending on the features included in the strategies used by each individual.

\section{Variability in Task Representations}

One of RCCL's main contributions is its focus on the role of task representations in choice learning. To describe the influence of representations on choice, RCCL introduces several features that lead to novel predictions. First, RCCL includes a process for selecting a subset of the possible task features to be included in the chooser's representation. Second, RCCL includes a process for generating choice strategies that depends on the task representation. Third, RCCL includes a process for changing the task representation according to the effectiveness of the current strategy set. In combination, these processes predict two prominent, observable sources of variation: (a) qualitative shifts in individuals' choice behavior across time and (b) distinct differences in choice behavior across participants (above and beyond sampling variation). In our original analysis of participants' choice behavior in two different tasks, we found substantial evidence for both kinds of variation, consistent with RCCL (Lovett \& Schunn, 1999). A nonrepresentational account such as that of Goodie and Fantino (1995, 1996, 1999), however, does not lead one to investigate such differences and, if they are found, does not fully account for them.

For example, in Goodie and Fantino (1995), as in our study, human participants were presented with many trials, each of which consisted of a cue (a green or blue square) followed by a binary choice. The two choices were a green square and a blue square, so each choice could be viewed as matching the cue or not matching the cue. With "correct/incorrect" feedback, participants were expected to learn which choice was associated with each cue. The two cues were differentially predictive of the correct choice (i.e., one cue was more reliable and one was less reliable). In one condition, the two cues were $80 \%$ and $50 \%$ reliable, and in another 


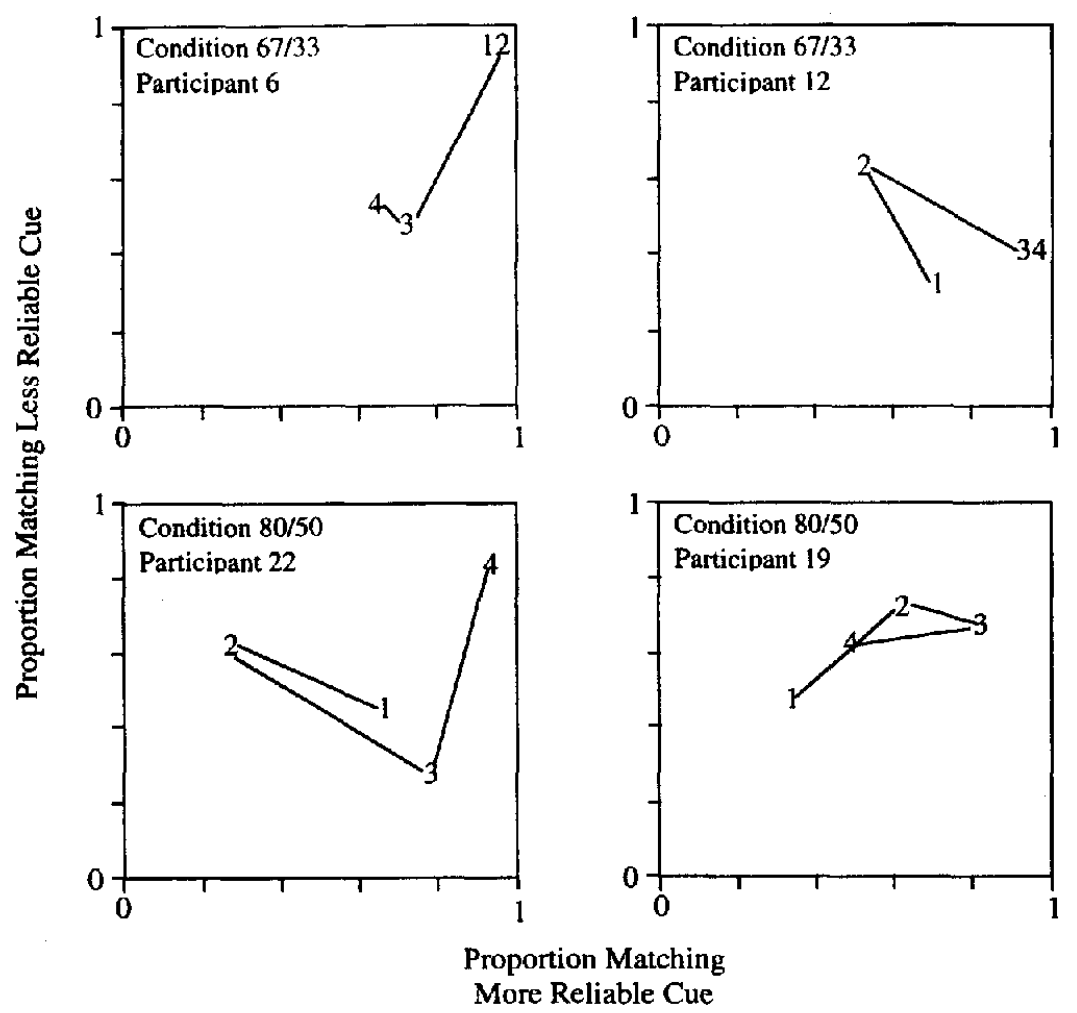

Figure 1. Individual choice trajectories for 4 participants from Goodie and Fantino (1995, Experiment 1). Numbers 1-4 indicate choice behavior in the first through fourth block of trials for each participant. Behavior in a given block is described in terms of the proportion of matching choices to the more reliable cue ( $x$-axis) and proportion of matching choices to the less reliable cue (y-axis).

condition they were $67 \%$ and $33 \%$ reliable. ${ }^{1}$ Goodie and Fantino (1995) concluded that participants' choices did not adequately differentiate between the cues because, for both conditions, the group's average choice proportions under the two cues were only slightly different. Goodie and Fantino did not, however, remark upon the systematic source of individual differences across participants or the qualitative changes in individual participants' performance across time. ${ }^{2}$

We reanalyzed their original data $^{3}$ and found both kinds of variation, as we had in Lovett and Schunn (1999). For example, Figure 1 presents the time course of choice for 4 individual participants in Goodie and Fantino's (1995) Experiment 1. ${ }^{4}$ Each panel plots a single participant's choice behavior across four blocks of the experiment, denoted by the labels $1,2,3$, and $4 .^{5} \mathrm{~A}$ given block's behavior is described by two dimensions: the proportion of trials on which the participant matched the more reliable cue ( $x$-axis) and the proportion of trials on which the participant matched the less reliable cue (y-axis). Note that all of these participants show qualitative shifts in their choice behavior across time. For example, Participant 6 from Condition 67/33 exhibited a marked change between Blocks 2 and 3, going from exclusive matching for both cues to mostly matching for the more reliable cue and random matching for the less reliable cue. RCCL explains this change in behavior as the result of changes in the chooser's task representation and strategies; other accounts (including Goodie and Fantino's as we understand it) do not explain it.
It is also important to note that many of the individual participant-block data points in Figure 1 reveal choice performance that differentiates between the two cues (i.e., many labels lie off the main diagonal). Thus, our reanalysis contrasts with Goodie and Fantino's (1995) aggregate analyses that suggested participants' choices for the two cues were only slightly different,

\footnotetext{
${ }^{1}$ We label the first condition $80 / 50$ and the second condition $67 / 33$, whereas Goodie and Fantino (1995) use the average reliability across the two cue types: $67 \%$ for the first condition and $50 \%$ for the second condition.

${ }^{2}$ In their more recent work, Goodie and Fantino (1999) have made some mention of within subject variability; however, they do not provide an explanation of it.

${ }^{3}$ We would like to thank Adam Goodie for sharing these data.

${ }^{4}$ Although these participants were chosen to highlight several interesting patterns, they are representative of the sample. The same analysis of Experiment 2 data from Goodie and Fantino (1995) produced similar results.

${ }^{5}$ We divided this experiment into four blocks to capture the first half and second half of each of the two sessions. Because different participants completed different numbers of trials, we defined block size to be 40 trials or the maximum possible given each participant's session length. Other than the block sizes, this analysis of Goodie and Fantino's (1995) data mimics the individual participant analysis presented in Experiment 2 of Lovett and Schunn (1999).
} 
and it offers an example of the dangers of averaging over data (see also Estes, 1956; Maddox, 1999; Siegler, 1987).

\section{RCCL Is a Broad Framework}

RCCL was designed as a broad framework for analyzing and explaining the process by which people learn to make choices across a wide set of domains. A theoretical framework embodies a set of interpretable principles that specific models must instantiate (Marr, 1982). It serves as a foundation upon which to build specific computational models over a variety of domains. Indeed, we are currently working on computational models of the two issues highlighted by RCCL, variability and task representations (Lovett, Daily, \& Reder, 2000; Schunn, 1999).

We set forth a framework before building specific models in the case of base-rate learning for several reasons. First, the existing data on base-rate learning supported several distinct computational models, suggesting there was room for more data-driven constraint. Our work revealed a new phenomenon in base-rate learning (i.e., the presence of representational change and variability) that had not yet been explored. RCCL provides an explanatory framework for this phenomenon that can influence new computational theory development. Second, there was a recent empirical result that was incompatible with the existing computational models: Goodie and Fantino's (1995) finding of incomplete base-rate sensitivity in nontest trials of the experiential paradigm. The fact that existing models could not explain this result suggested a need for theoretical expansion. A coherent framework is one way to bring meaning to a diverse and apparently contradictory body of results.

Third, the process of constructing and testing a new framework raises a variety of important theoretical and empirical questions, for example, How do choosers' views of a task arise? How and under what conditions do people change their choice strategies? How do choosers learn to prefer better strategies? Addressing some of these questions leads to new lines of inquiry, as we have highlighted above. Others of these questions involve drawing on existing theories and integrating them (with potential adjustments) into the framework. Goodie and Fantino (2000) argue that RCCL's prediction about learning to prefer more successful strategies is simply a repetition of the Law of Effect (Thorndike, 1932) and thus need not be stated. Yet, even if we as researchers have strong prior beliefs as to how this question will be answered, it must still be addressed within a complete framework. Moreover, we should emphasize that RCCL does not merely restate the Law of Effect but rather extends it to apply to mental constructs, such as strategies and representational features, that were not part of Thorndike's thinking.

In a similar vein, Goodie and Fantino claim that RCCL's predictions regarding salience are underspecified. However, as we mention in Lovett and Schunn (1999), there are existing models on salience effects that make similar predictions. These models are specific computational models, whereas RCCL is a framework that can integrate them into a coherent whole. We view this unifying approach that builds upon and develops novel combinations of existing theoretical and empirical work not as a weakness, as Goodie and Fantino suggest, but as a strength. We should also note here that prior empirical results on the Building Sticks Task (Lovett \& Anderson, 1996) were incorporated into some of RCCL's salience predictions.

\section{Base-Rate Errors}

Several of the issues raised by Goodie and Fantino (2000) arise from a disagreement on the role of base rates in choice. Goodie and Fantino appear to take base rates as a feature of the task that choosers can include in their task representations. This assumes that choosers have access to the base rates of the task as a whole. Although such information is given directly in the word-problem paradigm (e.g., $80 \%$ of the taxicabs in the city are green), compiling it across trials in the experiential paradigm is nontrivial. (Indeed, the heart of the experiential paradigm involves processing trials one at a time.) It is not necessary to assume that choosers store all possible base rates of a task. Instead, by defining the task representation as "the set of stimulus features an individual uses to encode the task environment" (Lovett \& Schunn, 1999, p. 108; emphasis added) and generating choice strategies as particular combinations of these features, RCCL shows a way for base-rate information to influence behavior: The various success rates of the generated strategies automatically encode different kinds of baserate information; at the same time, these success rates influence people to choose the more successful strategies. Hence, RCCL posits that, by repeated use of the generated strategies, choosers learn and behave according to the base rates of success of those strategies (e.g., for the strategy "if the cue is a blue square, pick the left choice" choosers would learn the conditional probability of left choice given a blue cue; for the strategy "match the cue" choosers would learn the unconditional probability of matching). Depending on what features are included in the used strategies (e.g., color of the cue, match status of the choice), these strategy-wise base rates may or may not reflect the base rates of the two choice colors overall. As we found in Lovett and Schunn (1999), participants tend not to represent the choices in terms of their absolute colors but rather in terms of their matching status relative to the cue color.

Goodie and Fantino suggest that RCCL's predictions for Experiment 2 of Lovett and Schunn (1999) imply sensitivity to the base rates of the two choice colors. As just explained, this is not so. Rather, there are a variety of different base rates to which choosers could be sensitive (e.g., matching the cue/not matching the cue, green/blue, left/right). To people unfamiliar with Tversky and Kahneman's (1982) taxicab problem, emphasizing the base rates of the two choice colors is an arbitrary choice. According to RCCL, it is the chooser's task representation that determines which of the many possible base rates will affect choice behavior.

\section{Coda}

A key contribution of Lovett and Schunn (1999) was showing that important sources of variation were missing from previous accounts of base-rate learning. We explained this in terms of task representations that can change over time and differ between people. RCCL was designed to embody several principles for how to think about task representations and as a platform for theoretical development of this novel component in learning to make choices.

\section{References}

Estes, W. K. (1956). The problem of inference from curves based on group data. Psychological Bulletin, 53, 134-140. 
Goodie, A. S., \& Fantino, E. (1995). An experientially derived base-rate error in humans. Psychological Science, 6, 101-106.

Goodie, A. S., \& Fantino, E. (1996). Learning to commit or avoid the base-rate error. Nature, 380, 247-249.

Goodie, A. S., \& Fantino, E. (1999). Base rates versus sample accuracy: Competition for control in human matching to sample. Journal of the Experimental Analysis of Behavior, 71, 155-169.

Goodie, A. S., \& Fantino, E. (2000). Representing the task in Bayesian reasoning: Comment on Lovett and Schunn (1999). Joumal of Experimental Psychology: General, 129, 449-452.

Lovett, M. C., \& Anderson, J. R. (1996). History of success and current context in problem solving: Combined influences on operator selection. Cognitive Psychology, 31, 168-217.

Lovett, M. C., Daily, L. Z., \& Reder, L. M. (2000). A source activation theory of working memory: Cross-task prediction of performance in ACT-R. Cognitive Systems Research, 1, 99-118.

Lovett, M. C., \& Schunn, C. D. (1999). Task representations, strategy variability, and base-rate neglect. Journal of Experimental Psychology: General, 128, 107-130.

Maddox, W. T. (1999). On the dangers of averaging across observers when comparing decision bound models and generalized context models of categorization. Perception \& Psychophysics, 61(2), 354-375.

Marr, D. (1982). Vision. San Francisco: W. H. Freeman.

Schunn, C. D. (1999, August). Exploring individual variability using ACT-R. Paper presented at the Sixth Annual ACT-R Workshop, Fairfax, VA.

Siegler, R. S. (1987). The perils of averaging data over strategies: An example from children's addition. Journal of Experimental Psychology: General, 116, 250-264.

Thorndike, E. L. (1932). The fundamentals of learning. New York: Columbia University Press.

Tversky, A., \& Kahneman, D. (1982). Evidential impact of base rates. In D. Kahneman, P. Slovic, \& A. Tversky (Eds.), Judgment under uncertainty: Heuristics and biases (pp. 153-160). New York: Cambridge University Press.

\section{New Editors Appointed, 2002-2007}

The Publications and Communications Board of the American Psychological Association announces the appointment of five new editors for 6-year terms beginning in 2002.

As of January 1, 2001, manuscripts should be directed as follows:

- For Behavioral Neuroscience, submit manuscripts to John F. Disterhoft, $\mathrm{PhD}$, Department of Cell and Molecular Biology, Northwestern University Medical School, 303 E. Chicago Avenue, Chicago, IL 60611-3008.

- For the Journal of Experimental Psychology: Applied, submit manuscripts to Phillip L. Ackerman, PhD, Georgia Institute of Technology, School of Psychology, MC 0170, 274 5th Street, Atlanta, GA 30332-0170.

- For the Journal of Experimental Psychology: General, submit manuscripts to D. Stephen Lindsay, PhD, Department of Psychology, University of Victoria, P.O. Box 3050, Victoria, British Columbia, Canada V8W 3P5.

- For Neuropsychology, submit manuscripts to James T. Becker, PhD, Neuropsychology Research Program, 3501 Forbes Avenue, Suite 830, Pittsburgh, PA 15213.

- For Psychological Methods, submit manuscripts to Stephen G. West, PhD, Department of Psychology, Arizona State University, Tempe, AZ 85287-1104.

Manuscript submission patterns make the precise date of completion of the 2001 volumes uncertain. Current editors, Michela Gallagher, PhD; Raymond S. Nickerson, PhD; Nora S. Newcombe, PhD; Patricia B. Sutker, PhD; and Mark I. Appelbaum, $\mathrm{PhD}$, respectively, will receive and consider manuscripts through December 31, 2000. Should 2001 volumes be completed before that date, manuscripts will be redirected to the new editors for consideration in 2002 volumes. 